\title{
Phase II trial of docetaxel, cisplatin and 5FU chemotherapy in locally advanced and metastatic penis cancer (CRUK/09/001)
}

\author{
S Nicholson *,1,11 , E Hall ${ }^{2,11}$, S J Harland ${ }^{3}$, J D Chester ${ }^{4,12}$, L Pickering ${ }^{5}$, J Barber ${ }^{6}$, T Elliott ${ }^{7}$, A Thomson $^{8}$,
} S Burnett ${ }^{2}$, C Cruickshank ${ }^{2}$, B Carrington ${ }^{9}$, R Waters $^{2}$ and A Bahl ${ }^{10}$

${ }^{1}$ Imperial College Healthcare NHS Trust, Department of Medical Oncology, Charing Cross Hospital, London W6 8RF, UK; ${ }^{2}$ The Institute of Cancer Research, Clinical Trials \& Statistics Unit, 123 Old Brompton Road, London SW7 3RP, UK; ${ }^{3}$ Department of Oncology, University College London Hospitals NHSFT, 250 Euston Road, London NW1 2PG, UK; ${ }^{4}$ Leeds Institute of Molecular Medicine, University of Leeds and St. James's Institute of Oncology, St James's University Hospital, Leeds LS9 7TF, UK; ${ }^{5}$ Department of Oncology, St. Georges Hospital, Blackshaw Road, Tooting, London SW17 OQT, UK; ${ }^{6}$ Velindre Cancer Centre, Velindre Hospital, Velindre Road, Whitchurch, Cardiff CF14 2TL, UK; ${ }^{7}$ Department of Clinical Oncology, The Christie Hospital, Wilmslow Road, Manchester M20 4BX, UK; ${ }^{8}$ Department of Oncology, Royal Cornwall Hospital, London Road, Treliske, Truro TR1 3LJ, UK; ${ }^{9}$ Department of Diagnostic Radiotherapy, The Christie Hospital, Manchester, UK and ${ }^{10}$ Bristol Haematology and Oncology Centre, Horfield Road, Bristol BS2 8ED, UK

Background: Penis cancer is rare and clinical trial evidence on which to base treatment decisions is limited. Case reports suggest that the combination of docetaxel, cisplatin and 5-flurouracil (TPF) is highly active in this disease.

Methods: Twenty-nine patients with locally advanced or metastatic squamous carcinoma of the penis were recruited into a single-arm phase II trial from nine UK centres. Up to three cycles of chemotherapy were received (docetaxel $75 \mathrm{mg} \mathrm{m}^{-2}$ day 1 , cisplatin $60 \mathrm{mg} \mathrm{m}^{-2}$ day 1,5 -flurouracil $750 \mathrm{mg} \mathrm{m}^{-2}$ per day days 1-5, repeated every 3 weeks). Primary outcome was objective response (assessed by RECIST). Fourteen or more responses in 26 evaluable patients were required to confirm a response rate of $60 \%$ or higher (Fleming-A'Hern design), warranting further evaluation. Secondary endpoints included toxicity and survival.

Results: 10/26 evaluable patients (38.5\%, 95\% Cl: 20.2-59.4) achieved an objective response. Two patients with locally advanced disease achieved radiological complete remission. $65.5 \%$ of patients experienced at least one grade 3/4 adverse event.

Conclusion: Docetaxel, cisplatin and 5FU did not reach the pre-determined threshold for further research and caused significant toxicity. Our results do not support the routine use of TPF. The observed complete responses support further investigation of combination chemotherapy in the neoadjuvant setting.

Penis cancer is rare in Europe and North America. Annual incidence in the UK is 1.3 cases/100 000 men, with 515 new cases and 92 deaths in 2010 (Cancer Research UK, 2013). This rarity means there is a lack of prospective clinical trial data to guide management.
Chemotherapy for penis cancer is used as palliation of metastatic disease. It may also have a role as neoadjuvant treatment for locally advanced disease, but the rarity of the tumour hampers attempts to establish a solid evidence base for its routine use.

*Correspondence: Dr S Nicholson; E-mail: peniletpf-icrctsu@icr.ac.uk

${ }^{11}$ Joint first authors.

${ }^{12}$ Current address: Institute of Cancer and Genetics, School of Medicine, Cardiff University and Velindre Cancer Centre, Cardiff, UK.

Received 24 June 2013; revised 11 September 2013; accepted 15 September 2013;

published online 29 October 2013

(c) 2013 Cancer Research UK. All rights reserved 0007-0920/13




Single-agent response rate to cisplatin is 23\% (Sklaroff and Yagoda, 1979; Ahmed et al, 1984; Gagliano et al, 1989), which is not dissimilar to the overall response rate of $30.8 \%$ reported for irinotecan and cisplatin in the EORTC single-arm phase II trial (Theodore et al, 2008). The combination of cisplatin and 5-flurouracil (PF) has been used to treat advanced squamous carcinoma of the penis since 1990 and is widely regarded as the standard of care in this setting. Three single-institution case series define its activity in a total of 19 patients, with a pooled response rate of $63 \%$ (three complete remissions and nine partial remissions) (Fisher et al, 1990; Hussein et al, 1990; Shammas et al, 1992). A recent retrospective series by Di Lorenzo et al (2012) suggests a response rate of $32 \%$ for this regimen, which is more in keeping with anecdotal experience.

The first report of a platinum-taxane combination in penis cancer describes a single patient with locally advanced disease who received carboplatin and paclitaxel (Joerger et al, 2004) and was rendered operable. Subsequent papers have described the use of the TIP regimen (cisplatin, paclitaxel and ifosfamide) in the neoadjuvant setting, confirming its ability to downstage locally advanced disease (Bermejo et al, 2007; Pagliaro et al, 2010).

The combination of docetaxel, cisplatin and 5FU (TPF) has produced high-response rates and improved survival outcomes compared with PF in squamous carcinomas of the head and neck (Posner et al, 2007; Vermorken et al, 2007), which shows histological and some clinical similarities to penis cancer. A single-institution retrospective case series describes the use of the TPF combination in six penis cancer patients with nodal disease: five patients responded, three with pathological complete remission (Pizzocaro et al, 2009). A further single-centre study (reported in abstract only) describes a collection of patients treated in the adjuvant, neoadjuvant and palliative settings with cisplatin, $5 \mathrm{FU}$ and either paclitaxel or docetaxel. One response is described in six patients with metastatic disease and seven responses in 12 patients treated in the neoadjuvant setting, which would suggest an objective response rate of around 44\% (Salvioni et al, 2011).

The penile cancer subgroup of the UK National Cancer Research Institute's Bladder Cancer Clinical Studies Group designed a single-arm, multicentre phase II trial to establish the objective response rate of the TPF combination in penis cancer. Our aims also included establishing a network of centres to facilitate clinical trials in penis cancer.

\section{MATERIALS AND METHODS}

This study (CRUK/09/001, ISRCTN 78108737 (controlled-trials. com)) was co-sponsored by the Institute of Cancer Research and University Hospitals of Bristol NHS Foundation Trust. It was conducted in accordance with the principles of Good Clinical Practice and approved by the Medicines and Healthcare Products Regulatory Authority and London-Central Multicentre Research Ethics Committee. It was overseen by independent Trial Steering and Data Monitoring Committees.

Patients aged 18 years and over with histologically proven squamous carcinoma of the penis were required to have measurable disease (RECIST version 1, modified to RECIST 1.1 by protocol amendment, November 2009) staged (TNM classification version 6, (Sobin and Wittekind, 2002)) as follows: M1; or M0, any $\mathrm{T}$, N3 (that is, involvement of deep inguinal or pelvic lymph nodes); or M0, any T, N2 (that is, involvement of multiple or bilateral superficial lymph nodes); or M0, T3, N1 (tumour invades urethra or prostate and single inguinal lymph node involved); or M0, T4 (tumour invades other adjacent structures) any N.

Main eligibility criteria were glomerular filtration rate (GFR) of $60 \mathrm{ml} / \mathrm{min}$ or greater (subsequently modified to allow a GFR of
55-60 $\mathrm{ml}$ per min, subject to agreement by the Chief Investigator) and ECOG performance status 0 to 2 .

Exclusion criteria included non-squamous cancer of the penis, primary squamous carcinoma of the urethra, previous chemotherapy or radiotherapy, and prior malignancy (other than squamous cell carcinoma or basal cell carcinoma of non-penile skin) in the previous 5 years. All patients were required to give written informed consent.

Trial treatment consisted of three cycles of TPF with a cycle time of 21 days. Treatment comprised docetaxel $75 \mathrm{mg} \mathrm{m}^{-2}$ day 1 intravenously (i.v.) over $1 \mathrm{~h}$, cisplatin $60 \mathrm{mg} \mathrm{m}^{-2}$ day 1 i.v. over $2 \mathrm{~h}$ (preceded and followed by sodium chloride $0.9 \%$ over $1-2 \mathrm{~h}$ with potassium and magnesium supplementation according to local practice), followed by 5 -fluorouracil $750 \mathrm{mg} \mathrm{m}^{-2}$ per day as a continuous i.v. infusion over days $1-5$ (total dose $3750 \mathrm{mg} \mathrm{m}^{-2}$ ). The use of prophylactic granulocyte-colony stimulating factor was initially left to local investigator discretion, but an amendment to the protocol in October 2010 required all patients to receive prophylactic growth factor support at each cycle of chemotherapy. A single reduction in the dose of docetaxel (to $60 \mathrm{mg} \mathrm{m}^{-2}$ ) was permitted for toxicity, but no changes in dosage were permitted for cisplatin or 5FU. All patients required steroid premedication before docetaxel, and antiemetic therapy with steroids, $5 \mathrm{HT}_{3}$-antagonist and dopamine receptor $\mathrm{D}_{2}$-antagonist: doses and drugs used were in accordance with existing local policies for highly-emetogenic, docetaxel-based regimens.

Subsequent (post-trial) treatment was at local investigator discretion. Options included further cycles of TPF (for responding patients), chemoradiotherapy and surgery.

All patients underwent pre-treatment radiological assessment consisting of chest X-ray, CT scan of chest, abdomen and pelvis, and MRI of the pelvis and penis. In addition, all cutaneous and penile lesions were photographed.

Patients were assessed clinically before each cycle of treatment, on completion of the third cycle, and after formal radiological re-staging investigations. Clinical and radiological assessments were scheduled for 3, 6, 9, 12, 18 and 24 months after completion of study treatment.

The primary endpoint was radiological objective response rate (complete response plus partial response as defined by RECIST criteria) at 4 weeks from the date of commencement of the final cycle of study chemotherapy, that is, after a maximum of three cycles. CT scans were subject to central independent review by a single radiologist. Where MRI was used for follow-up imaging, these scans were also reviewed centrally.

Secondary endpoints included: the proportion of patients with inoperable loco-regional disease rendered operable by TPF chemotherapy; toxicity; progression-free survival (PFS) and overall survival (OS). Operability for all patients was assessed by each patient's supra-network multidisciplinary team and was defined as any previously-inoperable patient considered by the supra-network multidisciplinary team to be suitable to proceed to surgery after study treatment. Acute toxicity was graded according to the Common Toxicity Criteria for Adverse Events (CTCAE) version 3.0 after each cycle and at 3 months after the end of study treatment. Late toxicity (measured from 6 months to 2 years after end of treatment) will be reported on completion of follow-up.

This was a single-arm phase II study. An objective response rate of $60 \%$ or greater was regarded as necessary to merit further investigation of this regimen in this rare setting (with the allocation of resources that this would entail), while a response rate of $35 \%$ or lower would not be sufficient to warrant further research. A Fleming-A'Hern exact single-stage design (A'Hern, 2001) was used $(p 0=0.35, p 1=0.6)$ with one-sided $\alpha$ of 0.05 and $80 \%$ power. This required 26 evaluable patients, with 14 or more responses to be observed for further research to be considered feasible. It was envisaged that recruitment would take 18-24 months. 
Analysis of response was based on the evaluable population (26 patients). Patients were 'non-evaluable' if (i) they received less than 1 cycle of treatment before disease progression or penile cancer-related death or (ii) if disease was not measured at the end of treatment, either because the patient withdrew from trial for a reason unrelated to the treatment, or because the patient died on trial from other causes. Evaluability was reviewed by the Trial Management Group without knowledge of response outcome.

Sensitivity analyses were performed on all 29 enrolled patients and on a 'per-protocol' population of 26 patients. The 'perprotocol' population is defined as all enrolled patients who received at least one cycle of protocol chemotherapy without major protocol violations. This excluded two patients who were ineligible, and one who withdrew early from the study. These analyses classified patients as non-responders where response could not be assessed according to RECIST (for example, those dying before or refusing end-of-treatment re-staging assessments). Analyses of secondary outcomes used the enrolled population, with sensitivity analyses in the per-protocol population. Side effects experienced by $5 \%$ or more patients are reported.

Overall survival was defined as the time from enrolment until death from any cause. Progression-free survival was defined as time from enrolment until clinically or radiologically documented disease progression or death from any cause. Patients alive and/or progression-free at time of analysis or lost to follow-up were censored at date last seen. Kaplan-Meier estimates of PFS and OS in all patients and in locally advanced and metastatic patients separately are provided with $95 \%$ confidence intervals.

Patients were enrolled at the Cancer Research UK funded Clinical Trials and Statistics Unit at the Institute of Cancer Research (ICRCTSU), Sutton, Surrey, UK, where central trial management and all statistical analyses were performed. Analyses were based on a snapshot of the data taken on 30/11/2011 and were conducted using STATA 11.2 (StataCorp LP, College Station, TX, USA).

\section{RESULTS}

Twenty-nine patients were recruited from nine UK centres between September 2009 and December 2010; 26 patients were evaluable for response (Figure 1). Three patients were not evaluable for response: one died from progressive disease, having failed to complete one cycle of treatment (that is, $<21$ days after commencing trial treatment), one patient missed the end of treatment visit and therefore no disease measurements were

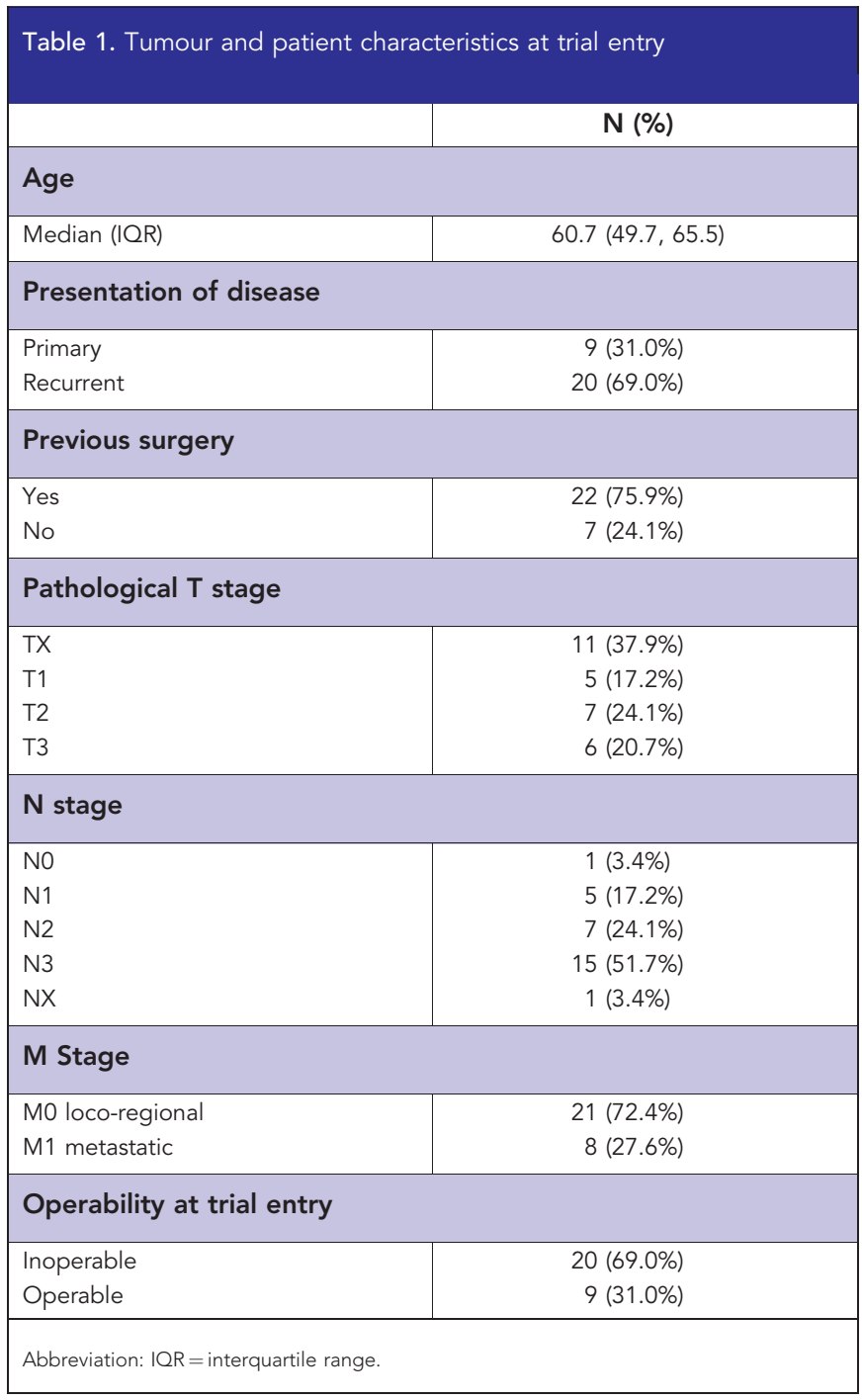

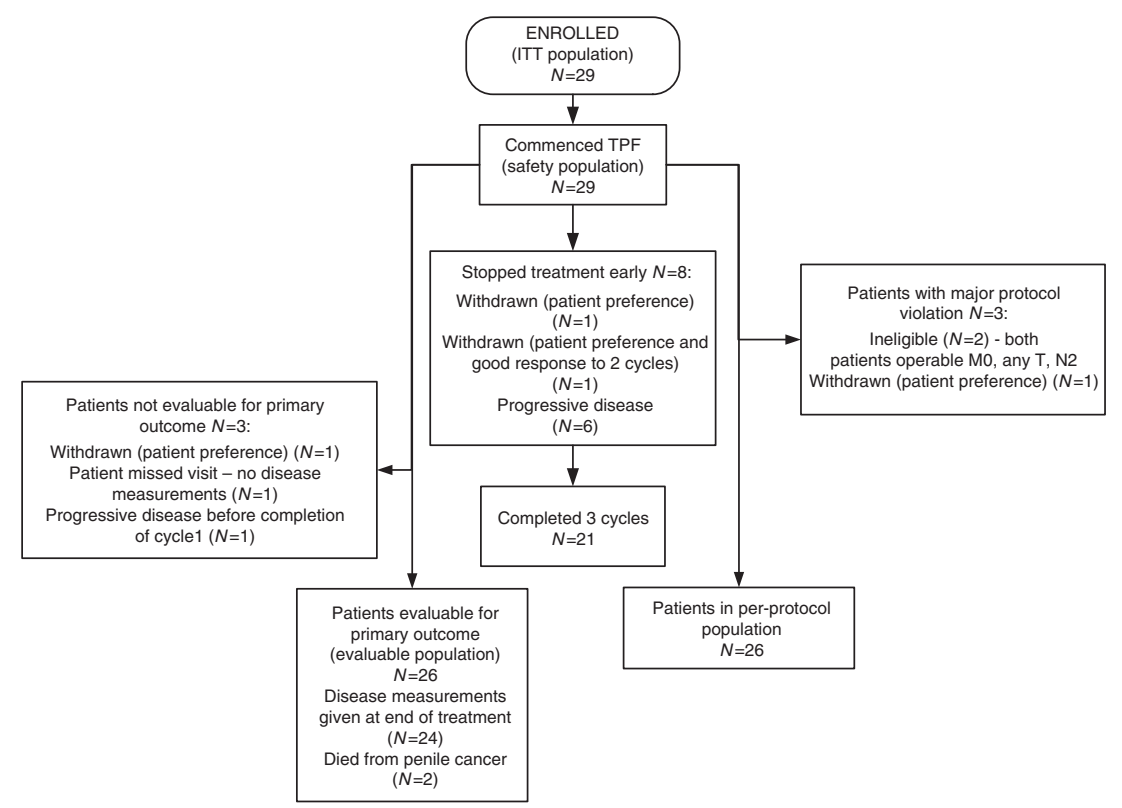

Figure 1. Patient flow through the trial. 
available and one withdrew from the trial. Tumour and patient characteristics are given in Table 1.

Overall mean relative dose intensity was $84 \%$. Twenty-one (72.4\%) patients received all three cycles of chemotherapy. Five patients (17.2\%) discontinued after two cycles, and three patients (10.3\%, all non-evaluable) after a single cycle. Eighteen patients had a dose reduction or delay; five due to toxicity, eight due to clinical decision, one through patient choice, four due to administrative error. Four dose reductions (docetaxel dose reduced from 75 to $60 \mathrm{mg} \mathrm{m}^{-2}$ ) resulted from haematological toxicity.

Objective responses were seen in 10 patients (two complete and eight partial remissions) resulting in a response rate of $38.5 \%$ (95\% CI: 20.2-59.4\%) (Table 2). Responses were seen in seven of 19 evaluable patients with locally advanced disease (OR: $36.8 \%$ (95\% CI: $16.3-61.6 \%)$ ) and in three of seven assessable patients with distant metastases (OR 42.9\%; (95\% CI: 9.9-81.6\%)).

Twenty patients were inoperable at trial entry, five $(25.0 \%$ (95\% CI: 8.6-49.1\%)) of whom were deemed operable after TPF chemotherapy; one of these was considered too frail to proceed to surgery.

Twenty-eight patients were assessable for toxicity (over a total of 76 cycles-Table 3). Nineteen patients $(67.9 \%$; $95 \%$ CI: 47.6-84.1\%) experienced one or more grade 3 or 4 toxicity during treatment. Sixteen of 28 (57.1\%), 10/26 (38.5\%) and 5/21 (23.8\%)

\begin{tabular}{|c|c|c|c|}
\hline Population & Responders & $\begin{array}{c}\text { Objective } \\
\text { response } \\
\text { rate } \\
(95 \% \mathrm{Cl})\end{array}$ & $95 \% \mathrm{Cl}$ \\
\hline Evaluable $(N=26)$ & 10 & $38.5 \%$ & $(20.2-59.4 \%)$ \\
\hline Locally advanced $(N=19)$ & 7 & $36.8 \%$ & $(16.3-61.6 \%)$ \\
\hline Metastatic $(N=7)$ & 3 & $42.9 \%$ & (9.9-81.6\%) \\
\hline $\begin{array}{l}\text { All enrolled ( } N=29, \\
\text { sensitivity analysis) }\end{array}$ & 10 & $34.5 \%$ & $(17.9-54.3 \%)$ \\
\hline $\begin{array}{l}\text { Per-Protocol ( } N=26 \text {, } \\
\text { sensitivity analysis) }\end{array}$ & 10 & $38.5 \%$ & $(20.2-59.4 \%)$ \\
\hline
\end{tabular}

Table 3. On-treatment toxicity (worst CTC grade reported over cycles 1 to 3 of treatment)

\begin{tabular}{|l|c|}
\hline Toxicity & $\begin{array}{c}\text { Number of patients } \\
\text { with grade 3/4 (\%) }\end{array}$ \\
\hline Any grade 3/4 toxicity & $19(65.5 \%)$ \\
\hline Neutropenia & $13(46.4 \%)$ \\
\hline Sepsis & $7(25.0 \%)$ \\
\hline Diarrhoea & $4(14.3 \%)$ \\
\hline Febrile neutropenia & $4(14.3 \%)$ \\
\hline Leucopenia & $4(14.3 \%)$ \\
\hline Anaemia & $2(7.1 \%)$ \\
\hline Asthenia & $2(7.1 \%)$ \\
\hline Nausea & $2(7.1 \%)$ \\
\hline Peripheral oedema & $2(7.1 \%)$ \\
\hline Syncope & $2(7.1 \%)$ \\
\hline Abbreviation: CTC=Common Toxicity Criteria. & \\
\hline
\end{tabular}

patients reporting at least one grade 3 or 4 toxicity after cycle 1,2 and 3 , respectively. The most common single grade 3 or 4 toxicity was neutropenia (13 patients; 46.4\%). Febrile neutropenia or neutropenic sepsis (grade 3 or 4 ) complicated $9.5 \%$ of cycles and were experienced by 6 patients (21.4\%). Three of $19(15.8 \%)$ patients reported grade 3 or 4 toxicity at 3 months ( 2 peripheral oedema; 1 hypertension). There were no toxic deaths and no suspected unexpected serious adverse reactions.

After a median follow-up of 14.5 months, 17/29 patients (58.6\%) had progressed and 15 (51.7\%) had died. Median PFS and OS for all enrolled patients, was 7.1 months (95\% CI: 2.7-upper limit not reached) and 13.9 months (6.1 - upper limit not reached), respectively. Median PFS and OS for the per-protocol population ( $n=26$, sensitivity analysis) are 6.9 and 7.7 months, respectively. Progression-free survival at 1 year for locally advanced and metastatic patients were $48.8 \%(25.0-68.9 \%)$ and $12.5 \%$ $(0.7-42.3 \%)$, respectively (Figure 2 ).

\section{DISCUSSION}

The incorporation of taxanes into chemotherapy for penile cancer is a recent phenomenon, and claims for added activity are not all based on good quality evidence. We set out to assess the efficacy of the docetaxel-containing TPF regimen in the management of this rare disease. The high-response rates reported in small, retrospective case series were not borne out in this prospective, multicenter phase II trial. Ten of 26 patients $(38.5 \%$; $95 \%$ CI: 20.2-59.4) responded, that is, less than the 14 required to recommend further research. Together these data do not support the routine use of TPF in either the palliative or the neoadjuvant setting. The small sample sizes and the observed complete responses in some patients may justify the use of complex


Figure 2. Progression-free survival (A) and overall survival (B) in months since trial entry. 
chemotherapy for some patients in the neoadjuvant setting. The high rate of toxicity militates against its use in the palliative setting.

The issue of toxicity requires further reflection, as it represents a key consideration in the design of future trials. The overall burden of toxicity is not always best represented by reporting of only grade 3 and 4 events: diarrhoea of all grades was experienced by $100 \%$ of patients in this trial, and this would very likely limit uptake of even an efficacious regimen in the palliative setting. The potential for improved dose delivery in the neoadjuvant setting (which might be a valid strategy to improve response rate) is hampered by the observations that more than $2 / 3$ of patients experienced at least one toxic event of grade 3 or 4 , while one-in-five experienced febrile neutropenia/neutropenic sepsis. It is probable that some of this toxicity could be ameliorated by dose reduction, but this would likely further compromise a response rate that is already insufficient to warrant further research.

The study has two main limitations. First, it was nonrandomised and any inference about the effect of adding docetaxel to standard cisplatin and 5FU is therefore based on historical data. The single-arm design was determined by the rarity of the disease. The exceptional speed of recruitment leads us to conclude that randomised trials may be possible, particularly with wider collaboration. Second, patients with both metastatic and locally advanced disease were included. This facilitated a trial with a simple primary endpoint (response rate) but precluded examination with any subtlety of the differing requirements for palliative and neoadjuvant treatment. The clear requirement for the latter is a sufficiently high-response rate, and this does not seem to apply to the TPF regimen (objective response rate $36.8 \%$ ) in the way that has been demonstrated by others (in a prospective trial) for the TIP regimen, with an objective response rate of 50\% (Pagliaro et al, 2010). Appropriate endpoints for the palliative setting are clinical benefit (objective response plus disease stabilisation rate), OS and toxicity, with symptomatic benefit rather harder to establish. The clinical benefit rate observed in the metastatic group (crudely 18/26 for all patients, $4 / 7$ metastatic patients) should be viewed in the light of the high rates of toxicity. Median OS of 13.9 months reflects the high proportion of patients with locally advanced disease, many of whom went on to receive consolidation or salvage with surgery, radiotherapy or both and indeed this (and rate of stable disease) may explain, at least in part, the apparent discrepancy between observed response and 1-year PFS rates ( $36.8 \%$ and $48.8 \%$, respectively) in the locally advanced subgroup. This encouraging figure supports the general principle that chemotherapy per se may have a role to have in the management of penis cancer, as exemplified by the two patients (both with locally advanced disease) who achieved complete remission on chemotherapy. The relative contribution of surgery and neoadjuvant chemotherapy to outcome in the four inoperable patients who were rendered operable by TPF and underwent surgery is difficult to ascertain, the numbers here being undeniably small. Surgery remains the mainstay of treatment for patients with locally advanced disease, and the place of neoadjuvant therapy in such patients will hopefully be defined in future trials.

The decision to conduct a national prospective clinical trial in cancer of the penis was facilitated by the reorganisation of cancer services in the UK. This process mandated that rarer tumours should be treated by supra-network multidisciplinary teams, comprising both surgical and non-surgical oncologists, specialist pathology, radiology and nursing input. Penis cancer in the UK is now treated in nine defined specialist centres, and this, in turn, has made rapid trial recruitment a reality.

The TPF trial recruited 29 patients in 15 months. The only other recent trial of similar size in this disease took over 8 years to recruit 30 patients (Pagliaro et al, 2010). This experience suggests that we are now in a position to conduct much larger studies within a manageable timeframe, and to address key questions of both routine management and novel therapeutics. The feasibility of such studies is likely to be facilitated by the International Rare Cancer Initiative, a collaboration between the US National Cancer Institute, the EORTC, the UK NIHR Cancer Research Network and Cancer Research UK.

\section{ACKNOWLEDGEMENTS}

We thank our patients, the investigators and the research support staff at all participating centres: Amit Bahl, Bristol Haematology and Oncology Centre (seven patients); Jim Barber, Velindre Hospital, Cardiff (one patient), John Chester, St James's University Hospital, Leeds (two patients); Tony Elliott, The Christie Hospital, Manchester (two patients); Stephen Harland, University College London Hospital (six patients); Helen Innes to October 2010, Shaun Tolan since October 2010, Clatterbridge Centre for Oncology (one patient), Steve Nicholson, Leicester Royal Infirmary, (five patients); Lisa Pickering, St George's Hospital, London (three patients); Alastair Thomson, Royal Cornwall Hospital (two patients). We also thank Eleftheria Kalaitzaki (ICR-CTSU) for her statistical input. Central trial management costs were funded by Cancer Research UK (C8262/A9765). Excess service costs were met by the UK's Comprehensive Local Research Networks. Partial funding was provided by Sanofi, as were drug supplies of docetaxel. Trial recruitment was facilitated within centres by the National Institute for Health Research (NIHR) Cancer Research Network.

\section{CONFLICT OF INTEREST}

A Bahl has participated on a Sanofi Aventis advisory board. L Pickering, T Elliott and S Harland declared travel Grants from Sanofi Aventis. All the remaining authors declare no conflict of interest.

\section{REFERENCES}

A'Hern R (2001) Sample size tables for exact single stage Phase II designs. Stat Med 20: 859-866.

Ahmed T, Sklaroff R, Yagoda A (1984) Sequential trials of methotrexate, cisplatin and bleomycin for penile cancer. J Urol 132(3): 465-468.

Bermejo C, Busby JE, Spiess PE, Heller L, Pagliaro LC, Pettaway CA (2007) Neoadjuvant chemotherapy followed by aggressive surgical consolidation for metastatic penile squamous cell carcinoma. J Urol 177(4): 1335-1338.

Cancer Research UK (2013) UK Cancer Incidence (2010) by Country Summary, April.

Di Lorenzo G, Buonerba C, Federico P, Perdonà S, Aieta M, Rescigno $\mathrm{P}$, D'Aniello C, Puglia L, Petremolo A, Ferro M, Marinelli A, Palmieri G, Sonpavde G, Mirone V, De Placido S (2012) Cisplatin and 5-fluorouracil in inoperable, stage IV squamous cell carcinoma of the penis. BJU Int (2012) 110(11 Pt B): E661-E666.

Fisher H, Barada J, Horton J (1990) Neoadjuvant therapy with cisplatin and 5 -fluoruracil for stage III squamous cell carcinoma of the penis. Acta Oncol 27(53): A652.

Gagliano RG, Blumenstein BA, Crawford ED, Stephens RL, Coltman Jr CA, Costanzi JJ (1989) cis-Diamminedichloroplatinum in the treatment of advanced epidermoid carcinoma of the penis: a Southwest Oncology Group Study. J Urol 141(1): 66-67.

Hussein A, Benedetto P, Sridhar K (1990) Chemotherapy with cisplatin and 5 -fluorouracil for penile and urethral squamous cell carcinomas. Cancer 65(3): 433-438.

Joerger M, Warzinek T, Klaeser B, Kluckert JT, Schmid HP, Gillessen S (2004) Major tumour regression after paclitaxel and carboplatin polychemotherapy in a patient with advanced penile cancer. Urology 63(4): 778-780. 
Pagliaro LC, Williams DL, Daliani D, Williams MB, Osai W, Kincaid M, Wen S, Thall PF, Pettaway CA (2010) Neoadjuvant paclitaxel, ifosfamide, and cisplatin chemotherapy for metastatic penile cancer: a phase II study. J Clin Oncol 28(24): 3851-3857.

Pizzocaro G, Nicolai N, Milani A (2009) Taxanes in combination with cisplatin and fluorouracil for advanced penile cancer: preliminary results. Eur Urol 55(3): 546-551.

Posner MR, Hershock DM, Blajman CR, Mickiewicz E, Winquist E, Gorbounova V, Tjulandin S, Shin DM, Cullen K, Ervin TJ, Murphy BA, Raez LE, Cohen RB, Spaulding M, Tishler RB, Roth B, Viroglio Rdel C, Venkatesan V, Romanov I, Agarwala S, Harter KW, Dugan M, Cmelak A, Markoe AM, Read PW, Steinbrenner L, Colevas AD, Norris Jr CM, Haddad RI (2007) Cisplatin and Fluorouracil Alone or with Docetaxel in Head and Neck Cancer. N Engl J Med 357(17): 1705-1715.

Salvioni R, Nicolai N, Piva L, Catanzaro M, Torelli T, Biasoni D, Stagni S, Milani A, Necchi A (2011) Pilot study of cisplatin, 5-fluorouracil, and a taxane (TPF) for advanced squamous cell carcinoma (SCC) of the penis. J Clin Oncol 29(suppl): (abstr 4639).

Shammas FV, Ous S, Fossa SD (1992) Cisplatin and 5-fluorouracil in advanced cancer of the penis. J Urol 147(3): 630-632.
Sklaroff RB, Yagoda A (1979) Cis-diamminedichloride platinum II (DDP) in the treatment of penile carcinoma. Cancer 44(5): 1563-1565.

Sobin L, Wittekind C (2002) TNM Classification of Malignant Tumours. 6th edn, Wiley-Liss: New York, NY, USA.

Theodore C, Skoneczna I, Bodrogi I, Leahy M, Kerst JM, Collette L, Ven K, Marréaud S, Oliver RD (2008) A phase II multicentre study of irinotecan (CPT 11) in combination with cisplatin (CDDP) in metastatic or locally advanced penile carcinoma (EORTC PROTOCOL 30992). Ann Oncol 19(7): 1304-1307.

Vermorken JB, Remenar E, van Herpen C, Gorlia T, Mesia R, Degardin M, Stewart JS, Jelic S, Betka J, Preiss JH, van den Weyngaert D, Awada A, Cupissol D, Kienzer HR, Rey A, Desaunois I, Bernier J, Lefebvre JL (2007) Cisplatin, Fluorouracil, and Docetaxel in unresectable head and neck cancer. N Engl J Med 357(17): 1695-1704.

(c) (1) (2) This work is licensed under a Creative Commons Attribution-NonCommercial-ShareAlike 3.0 Unported License. To view a copy of this license, visit http://creativecommons. org/licenses/by-nc-sa/3.0/ 